\title{
CHAPTER 16
}

\section{New Perspectives and a Review of Progress}

\author{
HANS-JOACHIM LEWERENZ*a AND LAURENCE PETER*b \\ a Joint Center for Artificial Photosynthesis, California Institute of \\ Technology, 1200 E. California Blvd, Pasadena, CA 91125, USA; \\ ${ }^{\mathrm{b}}$ Department of Chemistry, University of Bath, Bath BA2 7AY, \\ United Kingdom \\ *Email: lewerenz@caltech.edu; 1.m.peter@bath.ac.uk
}

\subsection{Introduction}

This final chapter reviews a range of topics that could advance the field of lightinduced energy conversion, in particular, of photoelectrochemical approaches, beyond current research and development activities. This compilation represents a subjective view with data and results considered from the fields of photonics, electronics, electrochemistry and life sciences. Our view of the relevance of these topics to the content of this book is given in short notes, and suggestions are outlined about how to incorporate the concepts and findings into the next generation of solar fuel generating structures and devices. The chapter concludes with a brief survey of progress towards the ultimate goal of generating solar fuels.

\subsection{Advanced Photonics}

\subsubsection{Surface Plasmons}

The excitation of volume and surface plasmons is well known in metal physics, and the reader is referred to a review by Raether and references therein for

RSC Energy and Environment Series No. 9

Photoelectrochemical Water Splitting: Materials, Processes and Architectures

Edited by Hans-Joachim Lewerenz and Laurence Peter

(C) The Royal Society of Chemistry 2013

Published by the Royal Society of Chemistry, www.rsc.org 
further details. ${ }^{1}$ In simple terms, the collective electron interaction is based on the long-range part of the Coulomb interaction and determines substantially the optical properties of metals and semiconductors. ${ }^{2}$ The dispersion relations for volume and surface plasmons and for photons $(\omega=c K)$ are displayed in

Figure 16.1. Three main features can be distinguished: the branch starting at $\omega_{\mathrm{P}}$ denotes the dispersion of longitudinal volume plasmons, the branch starting at zero energy approximating the frequency $\omega_{\mathrm{P}} / \sqrt{ } 2$ denotes the surface plasmon dispersion. In the shaded region, density fluctuations are damped exponentially, but excitation by light is possible, as can be seen from the figure where the photon dispersion intersects with the damped modes. The volume plasmon dispersion can be deduced from the properties of a nearly free electron gas, where the effective mass $m^{*}$ contains the energy band structure terms. ${ }^{3}$ The volume plasmon resonance is given by

$$
\omega_{p}=\sqrt{\frac{n_{e} q^{2}}{\varepsilon_{0} m^{*}}}
$$

and its dispersion for small $K$ values is

$$
\omega=\omega_{p}\left(1+\frac{3 v_{F}^{2}}{10 \omega_{p}^{2}} K_{/ /}^{2}\right)
$$

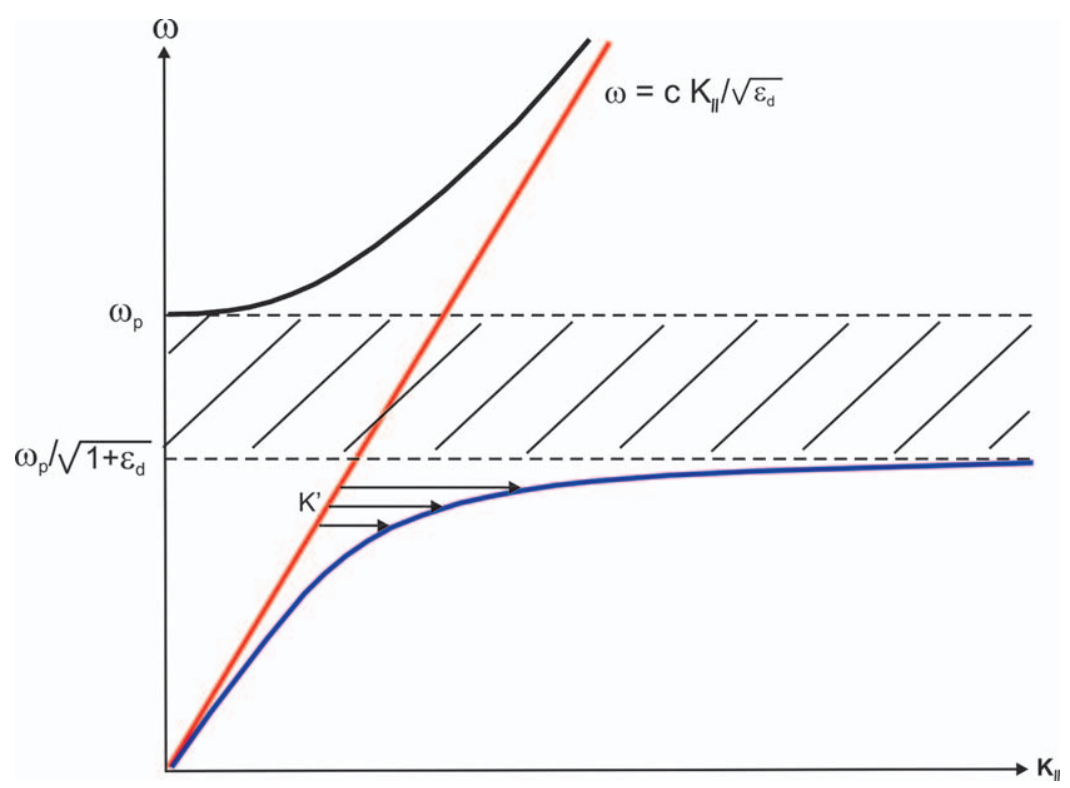

Figure 16.1 Dispersion relations for volume and surface plasmons of a nearly free electron gas; $c$, velocity of light; $K_{\|}$, wave vector parallel to the surface; $\omega_{\mathrm{p}}$, volume plasmon resonance frequency; $\varepsilon_{\mathrm{d}}$ permittivity of dielectric; the shaded region shows $\omega-K_{\|}$relations where no real solutions exist (damped modes); note the additional $K^{\prime}$ vectors from surface roughness that connect the light line to the surface plasmon dispersion (see text). 
The wave vector $K_{\|}$is parallel with the bounding surface, and one sees that additional $k$ vectors are needed to excite surface plasmons with light. These can be provided by surface roughness ${ }^{4}$ or by specific geometries.

The role of surface plasmons ${ }^{5}$ at the solid-electrolyte interface is an interesting topic, and in the present context one can ask whether surface plasmons can be used to induce photoelectrochemical reactions away from equilibrium conditions. First, the excitation and the decay of (delocalized) surface plasmons on electrochemically roughened Ag films is considered. In this case, the roughness adds a $K^{\prime}$-spectrum to the parallel wave vector dispersion that allows surface plasmon excitation, as indicated in Figure 16.1. A perfect test of the surface plasmon decay is provided by photoemission into electrolytes. ${ }^{6}$ This method allows one to investigate the energy relations of solids at photon energies below the vacuum work function, since the threshold for yield photoemission at the solid-acidic electrolyte contact is $\sim 3.1-3.2 \mathrm{eV}{ }^{7}$ Figure 16.2 shows the photoemission signal from the surface plasmon of roughened Ag (111), where the resonance energy lies at $3.5 \mathrm{eV}$, i.e. only slightly above the photoemission threshold. A distinct signal is noted at the surface plasmon energy. It follows that the surface plasmon can decay by emission of hot electrons with energy centered at the plasmon resonance energy. This is a first indication that such excitations can be used to induce photoelectrochemical reactions that otherwise need a large overpotential, such as $\mathrm{CO}_{2}$ reduction, where the first step requires an overpotential of $1.9 \mathrm{~V}{ }^{8}$ However, the quantum yield of electron emission is less than $10^{-3}$, which can be attributed to the following effects. Firstly, the emission cone for photoemission is very narrow for photoelectrons with kinetic energy only slightly above the threshold energy, and therefore a large fraction of the excited electrons will not be detected in the experiment (see Figure 16.3). Secondly, the generated roughness defines largely

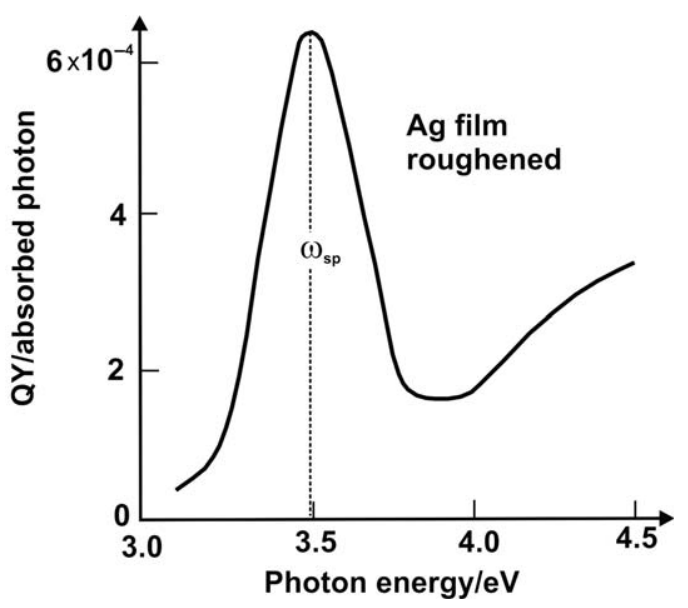

Figure 16.2 Photoemission-into-electrolyte spectrum of a roughened $\mathrm{Ag}$ film. Note the onset of the photoemission near $3.1 \mathrm{eV}$. Electrolyte, $1 \mathrm{~N}_{2} \mathrm{SO}_{4}$, counter electrode $\mathrm{Pt}$, reference electrode $\mathrm{NHE}\left(1 \mathrm{bar}_{2}\right)$ blackened $\mathrm{Pt}$; electrode potential $-0.2 \mathrm{~V}$ (NHE) (after reference 8 ). 


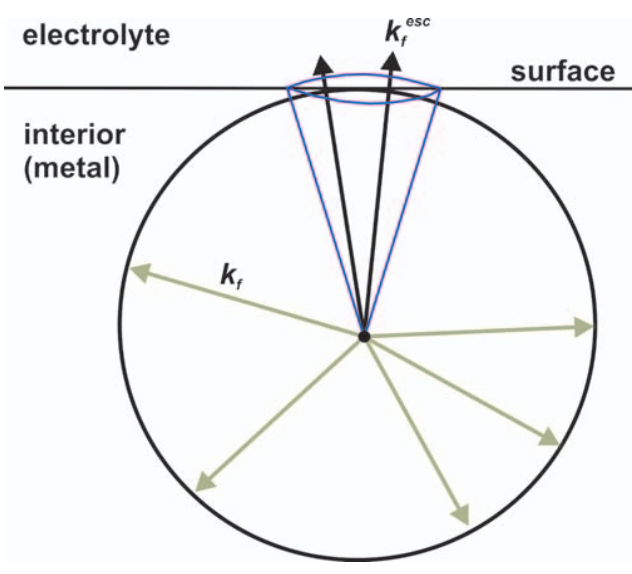

Figure 16.3 Schematic for the photoelectron emission cone in a yield experiment plotting the electron momenta in the final state, $k_{f}$, and indicating the surface (see text). The superscript 'esc' denotes the escaping photoelectrons that have sufficient momentum perpendicular to the surface to overcome the work function.

the spectrum of $K^{\prime}$ vectors, and from the photoemission experiment it was not possible to assess which roughness distribution would be particularly suited for a high yield of photoelectrons from surface plasmon decay.

The escape cone is defined by the electron momentum $\hbar \cdot k_{f}$ perpendicular to the surface, which is given by

$$
\left|k_{f}^{\perp}\right|=\frac{1}{\hbar} \sqrt{2 m^{*}\left(E_{f}-\Phi\right)}
$$

where the energetic difference between the final state energy $E_{\mathrm{f}}$ and the work function $\Phi$ determines the width of the escape cone:

$$
\theta=\cos ^{-1}\left(\frac{\left|k^{\perp}\right|}{|k|}\right)
$$

One observes that (i) hot electrons are injected into the electrolyte by surface plasmon decay and (ii) the quantum yield for the process is rather low, which can be related to the small energetic difference between the electron excess energy and the work function at the Ag-electrolyte contact. Therefore, in principle, it should be possible to induce reactions relatively far from equilibrium as shown in a schematic for two $\mathrm{CO}_{2}$ reduction steps in Figure 16.4. The first step occurs at $-1.9 \mathrm{~V} v s$. NHE and the second step (leading to the formate ion) at $-1.5 \mathrm{~V} .^{8}$

The role of localized surface plasmons in catalysis and electrochemistry has already been discussed to some extent. The resonance energy is typically lower than that of the surface plasmons shown in Figure 16.2, although it can be 


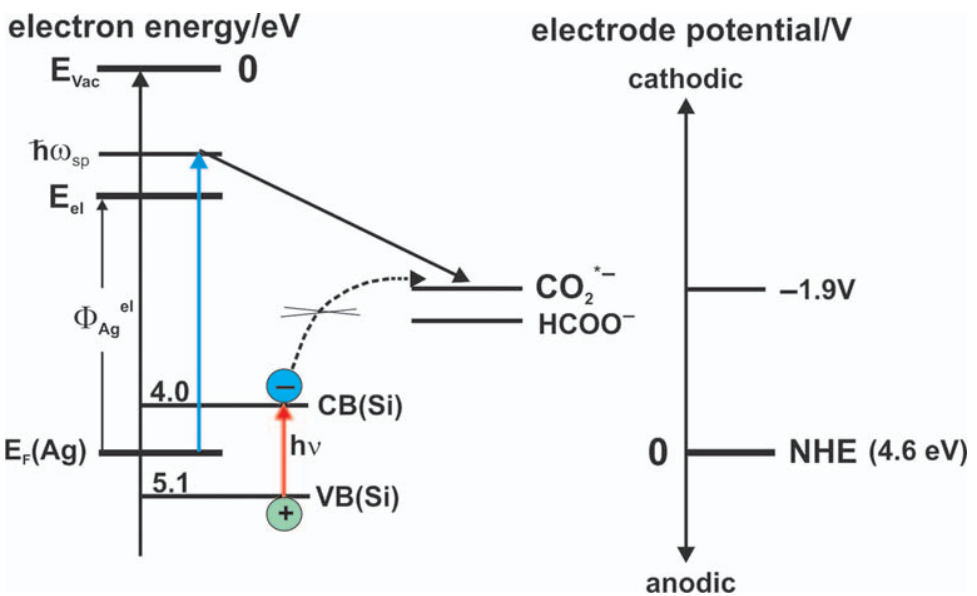

Figure 16.4 Energy and potential scheme for intermediate formation in carbon dioxide reduction; left: energy scheme related to the physics vacuum scale $\left(E_{\mathrm{Vac}}\right)$; the work function for $\mathrm{Ag}$ in acidic electrolyte, $\Phi_{\mathrm{Ag}}{ }^{\mathrm{el}}$, is labeled $E_{\mathrm{el}}$ and is approximately $3 \mathrm{eV}$. Also shown are the band edges of $\mathrm{Si}$ (electron affinity $\chi$ ) located at $4 \mathrm{eV}$ (conduction band, $\mathrm{CB}$ ) and at $5.1 \mathrm{eV}$ (valence band). The photoexcitation of $\mathrm{p}-\mathrm{Si}$ is shown to produce excess minority electrons that are not energetic enough to overcome the first step in $\mathrm{CO}_{2}$ reduction (note crossed dotted line); the excitation of a $\mathrm{Ag}$ surface plasmon (delocalized) can overcome the threshold. Also shown are the potentials for the $\mathrm{CO}_{2}{ }^{-}$radical and for $\mathrm{HCOO}^{-}$relative to NHE (see text).

tuned by size and shape of the nanoparticles that show the resonance. The resonance of localized surface plasmons (LSP) is found from the expression for the photon scattering cross section $\sigma_{\mathrm{SC}}$ in conjunction with the formula for the particle polarizability $\alpha$

$$
\begin{gathered}
\sigma_{S C} \propto\left(\frac{2 \pi}{\lambda_{p h}}\right)^{4}|\alpha|^{2} \text { or with } k_{p h}=\frac{2 \pi}{\lambda_{p h}}, \sigma_{S C}=k_{p h}^{4}|\alpha|^{2} \\
\alpha \propto V_{N P} \frac{\varepsilon_{N P}-\varepsilon_{M}}{\varepsilon_{N P}+2 \varepsilon_{M}}
\end{gathered}
$$

where the photon wavelength, the nanoparticle volume $V_{N P}$, and the permittivities of the metal nanoparticle and of the host, $\varepsilon_{N P}, \varepsilon_{M}$, respectively enter the expressions. A typical result of the enhancement of the absorbance of $\mathrm{Au}$ nanoparticles with different sizes is shown in Figure 16.5.

One also observes a small shift in the resonance frequency towards lower wavelength with decreasing size. The surface plasmon resonance is given by the Froehlich condition $\varepsilon_{N P}=-2 \varepsilon_{M}$. The resonance energy in Figure 16.5 is about $2.3 \mathrm{eV}$, which would be enough to induce non-equilibrium photoelectrochemical reactions. However, a direct effect based on LSPs has not yet been demonstrated. LSP excitation is also accompanied by a strong increase in the electrical field strength, ${ }^{9}$ a fact that has been used in application of surface 


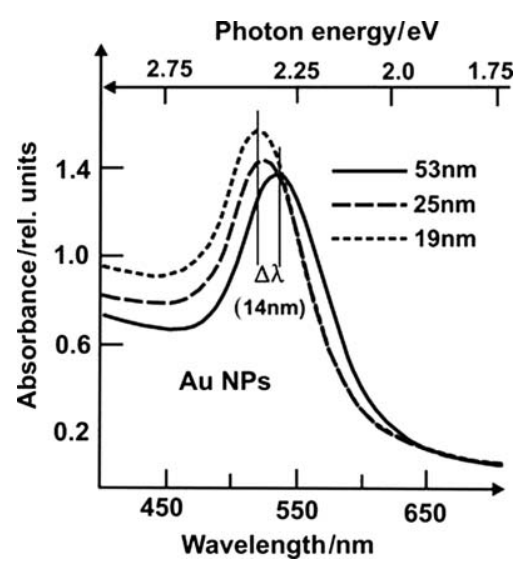

Figure 16.5 Spectral absorption of Au NPs of different sizes in the region near the LSP resonance (see text).

enhanced Raman scattering in biomedical sciences, for instance. ${ }^{10}$ It is also known that this resonance excitation results in temperature increases. ${ }^{11}$ Therefore, besides reactions involving energetic hot electrons, increases in reaction rates due to local heating and non-linear field effects might be envisaged. It will be difficult, however, to disentangle the contributions of the various photonic effects from the size- and shape dependence of the electrocatalytic activity of nanoparticles. Hitherto, convincing electrocatalysis based on LSP excitation has not yet been demonstrated, despite rather intense research in the field. ${ }^{12-16}$

\subsubsection{Coupled Förster Excitation Energy Transfer}

Carrier-free transfer of photonic excitation energy is known from photosynthesis, where the light absorption and the reactive centers are spatially separated. ${ }^{17}$ The transfer of the excitation energy to the reaction centers takes place in nature via multichromophoric Förster transfer, ${ }^{18,19}$ (see also chapter 14). In principle, this transfer follows the Hertzian dipole interaction and is described for a donor-acceptor molecular transfer by the transfer rate

$$
k_{T}=k_{D}\left(\frac{R_{0}}{R}\right)^{6}
$$

where the Förster radius $R_{0}$ is given by

$$
R_{0}^{6}=8.8 \cdot 10^{17} \frac{\kappa^{2}}{n^{4}} J
$$

In equations (16.5) and (16.6), $k_{D}$ denotes the donor fluorescence decay rate, $\kappa$ is the dipole orientation factor, $n$ the refractive index, and $J$ the overlap 
integral, given by the integral over energy of the product of the donor emission spectrum $F(v)$ and the acceptor absorption spectrum $A(v)$

$$
J=\int \frac{F(v) \cdot A(v)}{v^{4}} d v
$$

A simplified scheme showing the spectral overlap is presented in in Figure 16.6 for a single Förster transfer.

For photocatalytic reactions, it is of interest to know over what distance excitation energy can be transferred via the Förster mechanism. The Förster radius, $R_{0}$, is defined as the distance at which the energy transfer is $50 \%$ efficient. Typical values for $R_{0}$ lie in the range between 5 and $10 \mathrm{~nm}$. For Dexter energy transfer, this range is smaller, being $\sim 1 \mathrm{~nm} .{ }^{20}$ For the construction of a photoelectrocatalytic device where the location of absorption is clearly separated from that of the catalytic reaction (at a molecular catalyst for $\mathrm{CO}_{2}$ reduction, for instance), efficient operation would demand a highly porous surface texture, similar to that of dye sensitized solar cells. ${ }^{21,22}$ The complication and the challenge would be to achieve even distribution of absorption centers in very similar proximity (i.e. in the range of $5 \mathrm{~nm}$ ) to the catalyst surface. Although this goal might be achievable, it is more desirable to spatially separate light absorption and catalytically active sites, since then only catalyst stability is required if the remote absorber has no interface with the electrolyte. Non-radiative Förster transfer is limited to energy transfer over distances less

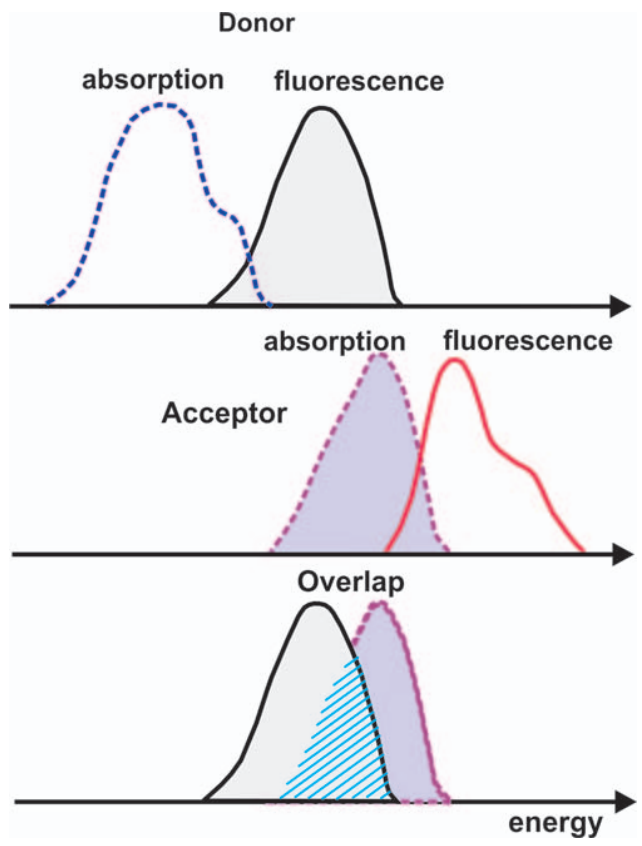

Figure 16.6 Schematic of the spectral overlap of donor fluorescence and acceptor absorption of a single molecule Förster transfer (see text). 
than $10 \mathrm{~nm}$, and it lacks controllable directionality. An approach that allows mitigation of these problems was introduced about a decade ago. ${ }^{23}$ It is based on a coupled Förster transfer - the surface plasmon polariton process. Surface plasmon polaritons (SPP) can be viewed as mixed electromagnetic-mechanical waves that propagate along interfaces between metals and dielectric media. Their propagation lengths can reach $100 \mu \mathrm{m}$. The coupling of the Förster process to SPP occurs via the coupling of the donor molecule dipole moment to the SPP mode. SPP excitation results in efficient dipole emission to acceptor molecules.

Figure 16.7 shows the arrangement for the coupling experiment. Donor and acceptor doped dielectric layers were separated by Ag films with varying thicknesses $d$ that are too large for classical Förster transfer. We select here $d=60 \mathrm{~nm}$. As donor dye, $\mathrm{Alq}_{3}$, dispersed in polymethylmetacrylate (PMMA) was used, and the acceptor dye was rhodamine 6Gin PMMA. The donor system was deposited onto silica, and Ag films were successively evaporated on the donor layer. The acceptor layer was deposited on top of the Ag layer.

Figure 16.8 shows a major result from the study for a $60 \mathrm{~nm}$ thick Ag film, where three spectra are displayed. The control spectra show the photoluminescence of the $\mathrm{Alq}_{3}$, which overlaps only weakly with the acceptor spectrum (blue line), and the directly excited emission from the R6G acceptor through the Ag film (red line). One sees that the donor control signal at $\lambda_{\text {res }}=520 \mathrm{~nm}$ is larger than that of the acceptor $\left(\lambda_{\text {res }}=565 \mathrm{~nm}\right)$. This is attributed to the fact that the excitation wavelength used $(408 \mathrm{~nm})$ matches the $\mathrm{Alq}_{3}$ absorption but is off resonance with the R6G absorption and, furthermore, that the acceptor signal is attenuated by the Ag film.

The black line in Figure 16.8 displays the characteristic features of the donor and acceptor. However, one observes that the emission from the R6G dye is enhanced by a factor of $\sim 10$ compared to the control sample (red line).

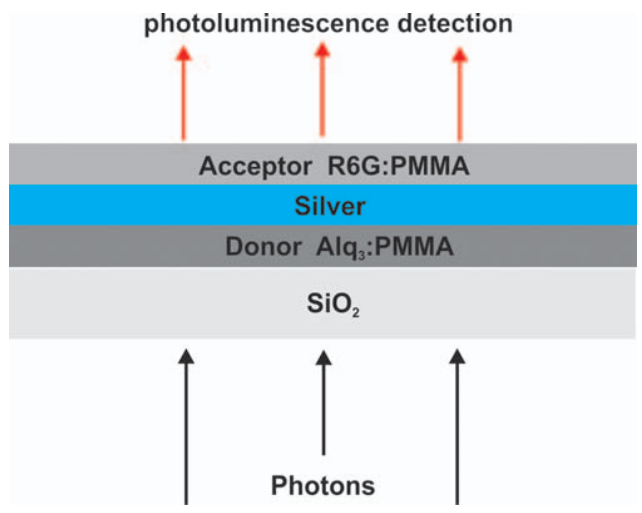

Figure 16.7 Experiment schematic for coupled Förster-SPP excitation energy transfer; laser excitation continuous wave diode laser, pump wavelength $408 \mathrm{~nm}$, i.e. minimum of acceptor absorption (R6G); PMMA thicknesses: $80 \mathrm{~nm}, \mathrm{Ag}$ thickness $60 \mathrm{~nm}$ (see text). 


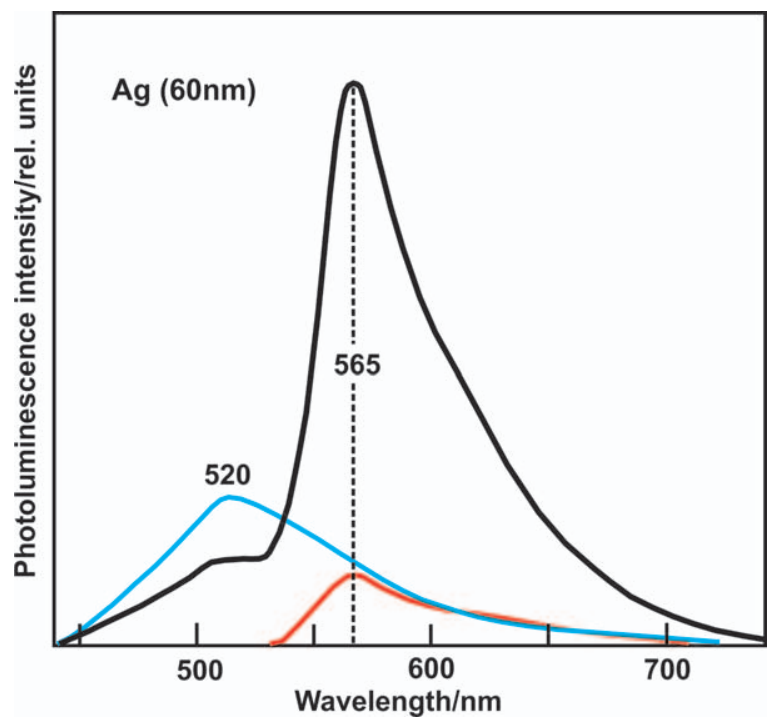

Figure 16.8 Photoluminescence spectra upon excitation of the structure shown in Figure 16.7 by laser light with $408 \mathrm{~nm}$ wavelength. Blue line, control spectrum for donor only, red line acceptor control spectrum, black line signal from samples with, both, donor and acceptor layers (see text).

This enhancement of the acceptor emission demonstrates that the excitation energy is transferred efficiently from the donor to the acceptor molecules by mediation of the Ag SPP over a distance that is much too large for classical Förster transfer. In principle, this effect could be used to design new architectures for synthetic light harvesting systems, where the SPP modes channel photonic excitation energy from light absorbing sites to catalytic reaction centers. So far, such designs have not yet been realized in practice.

\subsubsection{Lévy Processes}

In this subsection, the implications of the diffusive properties of light on energy conversion processes are summarized briefly, particularly in the context of the recent realization of a so-called Lévy glass. ${ }^{24,25}$ Models and theories of diffusion date back many years. They include the understanding of Brownian motion ${ }^{26}$ and Einstein's formulation in 1905 of Brownian random walk, actually used as proof for the existence of atoms. ${ }^{27,28}$ In optics, diffusion has proven to be a useful descriptor of systems in which randomly positioned particles scatter light in independent scattering events such that possible interference processes are cancelled out by the system's disorder. This "diffusion of light" was initially investigated in astrophysics in order to analyze interstellar light that has passed through dust and nebulae. ${ }^{29}$

Although light is described in classical electrodynamics as a wave, light scattering by inhomogeneities with multiple scattering paths can be described as 
a random walk process. The random walk picture allows one to describe, for instance, the propagation of light through opaque media such as clouds. If the successive scattering events inside such a medium are independent, the multiple scattering and the disorder smoothen out, resulting in isotropic Gaussian transport that manifests itself by the smooth white color of clouds of different altitudes and composition, although each water droplet in the clouds is transparent.

The fact that diverse phenomena such as heat-, sound- and light-diffusion can be described by Brownian random walk results from the Central Limit Theorem. ${ }^{30}$ This states that, for a large enough sample from a group of events (here light scattering) with a finite variance, the mean of all samples from that group will be approximately equal to the mean of the group of events and that all such samples show an approximate normal distribution pattern and all variances are approximately equal to the variance of the group of events divided by the size of each sample taken. In classical diffusion, the average squared displacement increases linearly with time

$$
\left\langle x^{2}\right\rangle=D \cdot t
$$

having the same form as the well-known relation for diffusion length $L$ of minority carriers in semiconductors. ${ }^{3}$

$$
L=\sqrt{D \cdot \tau}
$$

In systems that exhibit very strong fluctuations such as the spectral fluctuations in random lasers, the average step length is described by a power law $(\gamma>1)$

$$
\left\langle x^{2}\right\rangle=D \cdot t^{\gamma}
$$

indicating that extremely long jumps can occur and that the normal diffusion breaks down. This is the case for the so-called Lévy flights ${ }^{31}$ and has been termed superdiffusion, where the average squared displacement increases superlinearly with time. Classical diffusion is therefore a limiting case of Lévy flights. For $\gamma=2$, one has ballistic motion. Lévy random walks are a modification of the Lévy flights and, to compensate for the divergence of infinite jumps, long steps are penalized by coupling space and time. Classical and Lévy random walks are contrasted in Figure 16.9.

One sees that Lévy random walks are characterized by larger jumps and a type of loop near the end or beginning of the next jump. These features have been proven advantageous, for instance, in foraging. As another example, surface adsorbed polymers having a finite number of contact points with the surface can be modeled as a Lévy flight. As the figure shows, Lévy flights generally spread faster, leading to superdiffusion. Figure 16.10 compares the probability distribution functions for Gaussian and Lévy statistics. The latter is 
(a)

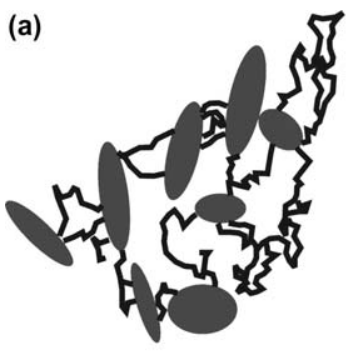

(b)

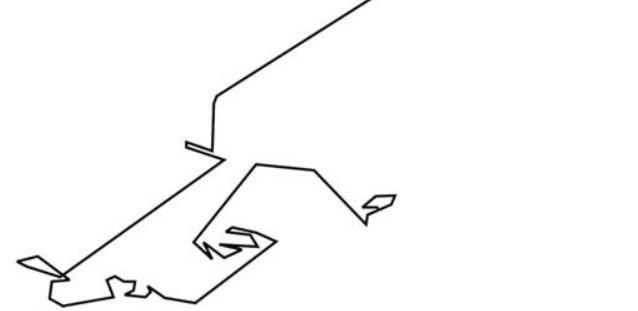

Figure 16.9 Schematic of classical Brownian walk (a) and of a Lévy random walk (b) for light scattering by a set of scattering elements with different sizes; in (a), the ellipsoidal and circular shapes indicate areas of high density of curling paths that are displayed for simplicity as filled areas; indeed, these areas are highly interconnected by random paths.

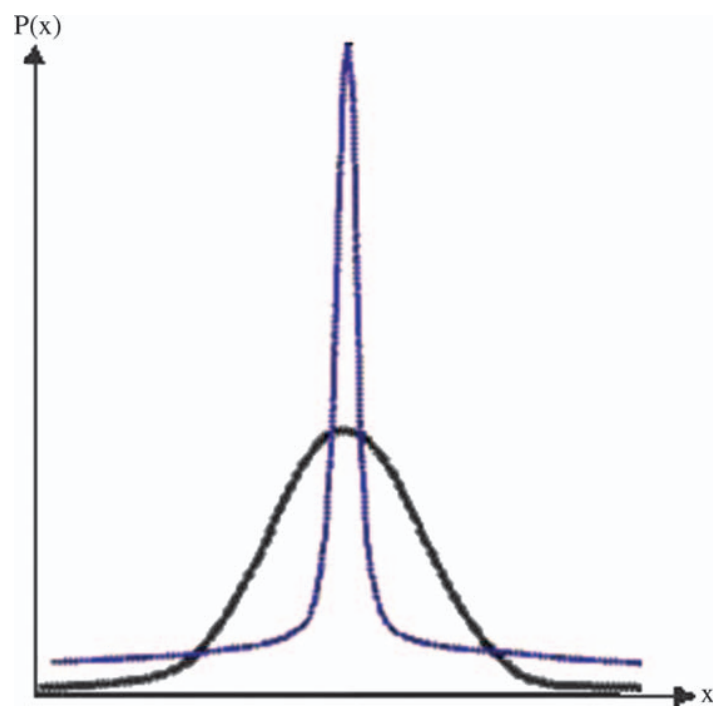

Figure 16.10 Comparison of the probability distribution function $P(x)$ of a Lévy flight (blue line) with that of a Gaussian distribution (black line) (see text).

characterized by a sharper and higher distribution near the center, i.e. stronger localization and, for $x$-values where the Gaussian distribution is $\sim 1 / 5^{\text {th }}$ of its maximum value, the Lévy tail becomes substantially larger compared to the 
Gauss distribution. The heavy tail (blue line in the figure) decays according to the inverse power law

$$
P(x)=\frac{1}{x^{\alpha+1}} \quad 1<\gamma \leq 2
$$

The value of $\alpha$ can be related to the superdiffusion coefficient $\gamma$ (see equation (16.10)): $\gamma=3-\alpha$ for $1 \leq \alpha<2$, hence $1<\gamma \leq 2$. For superdiffusion of light, this means that the transmission profile, $T(d)$ can appear as shown in Figure 16.11, which has been realized by the fabrication of a so-called Lévy glass. ${ }^{24,32}$ The heavy tail follows the form

$$
T \propto 1 / d^{\alpha / 2}
$$

where $d$ denotes the thickness of a slab-like sample, assuming that absorption is zero and only scattering occurs. $\alpha=2$ describes classical diffusive behavior, whereas superdiffusion occurs according to the above inequality for $\alpha$.

An intriguing aspect of superdiffusive light propagation is that the properties can be tuned by changing the parameters that determine the step length distribution via density variations arising from the chosen distribution of glass microsphere diameters. In the context of solar energy converting structures, the

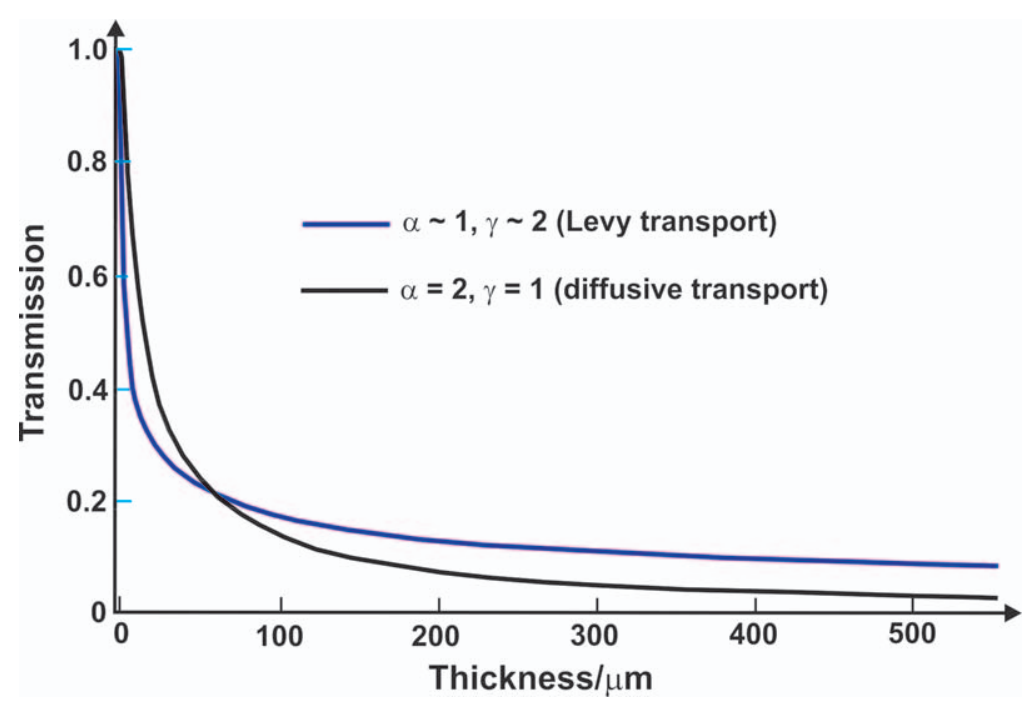

Figure 16.11 Total transmission $v s$. thickness for a fabricated Lévy glass with $\alpha \sim 1$ (corresponding to a Lorentzian Lévy flight-Cauchy distribution) compared to classical diffusive transport. The superdiffusive glass was fabricated by a suspension of titania nanoparticles in sodium silicate including an accurately chosen distribution of glass microspheres that have different diameters resulting in a modification of the density of scattering elements. The titania concentration was adjusted to achieve one scattering event occurring in the titania-filled spaces between adjacent glass microspheres (which themselves do not participate in scattering). 
possibility to design strongly altered and spatially extended excess minority carrier profiles is hitherto unexplored. A first approach in this direction is a process where the randomly moving particles are minority carriers of a semiconductor. ${ }^{33,34}$ The process, which is referred to as photon recycling, consists of photon assisted hopping of minority carriers as a result of radiative recombination that produces a photon that is re-absorbed at a distance via interband excitation. This presupposes that the material is a direct band gap semiconductor with a high rate of radiative recombination. The effect has been observed in moderately doped n-InP, where the reduced free carrier absorption ensures that the photon recycling process continues for $\sim 100$ times before a light generated minority hole recombines non-radiatively with a majority electron. It is the emission spectrum from the radiative recombination in combination with the probabilities for interband absorption and for photon propagation that produce the randomness of the process and which define the probability distribution of the walk of photons. Photons emitted near the absorption threshold of the semiconductor travel long distances before re-absorption takes place, resulting in the divergent variance typical for Lévy flights that leads to long-range Lévy walks for the excess carriers in semiconductors. Figure 16.12 shows the experimental arrangement for luminescence measurements and Figure 16.13 the results for a moderately doped $\mathrm{n}$-InP wafer.

It was found that the observed luminescence intensity was proportional to the intensity of the exciting light and that the spectral behavior of the luminescence was identical for all positions along the long axis of the slab. Based on comparison with model calculations, the proportionality of the local luminescence intensity with the excess carrier concentration could be shown. Accordingly, the observed intensity profile can be correlated to the local excess carrier concentration that is only a function perpendicular to the edge side.

Considering equation (16.10), where the average step length has been defined, the actual width of the distribution is described by $L \sim(D t)^{1 / \gamma^{*}}$ where $\gamma^{*}=0.5$ denotes classical diffusive transport $\left(L_{0}\right)$ as indicated in equation (16.9). For

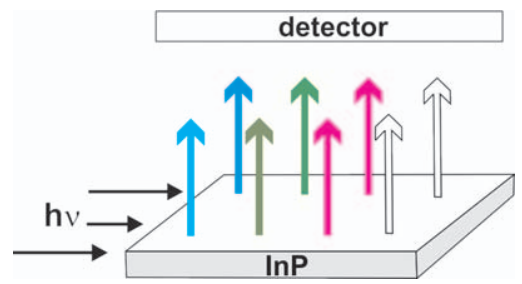

Figure 16.12 Experimental geometry for detection of long-range walks in a luminescence experiment; illumination: $808 \mathrm{~nm}$ laser (photon energy $1.53 \mathrm{eV}$, i.e. above the band gap energy of $\operatorname{InP}$ of $1.35 \mathrm{eV})$ at the $7 \mathrm{~mm}$ long edge face of an InP slab (length $20 \mathrm{~mm}$, thickness $350 \mu \mathrm{m}$ ). The luminescence spectra and luminescence intensity are detected perpendicular to the illumination direction as function of distance from the edge. The colored arrows mark different position from the edge. 


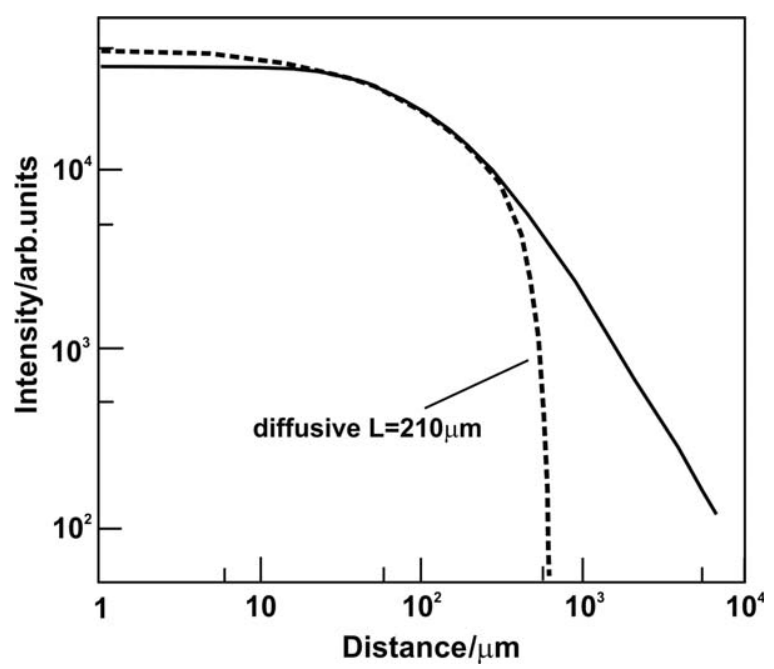

Figure 16.13 Luminescence intensity (excess carrier concentration) vs. distance from the illuminated edge side for moderately $\left(N_{\mathrm{D}}=3 \times 10^{17} \mathrm{~cm}^{-3}\right)$ doped n-InP plotted on a log-log scale; full line: experimental result; dashed line: calculation for classical diffusion assuming the untypically large diffusion length of $L=210 \mu \mathrm{m}$ (see text).

values $\gamma^{*}<2, L^{1 / \gamma^{*}} \gg L_{0}$. The tail of the solid line in Figure 16.13 is described by an exponent $\gamma^{*}=0.7$, which corresponds to a very pronounced spread beyond that expected for classical diffusion. The data thus demonstrate that, under specific circumstances, one can generate excess minority carriers much further away than the classical diffusion length from the light absorption profile. An initial interesting approach would be to analyze carefully the optical properties of nanoparticulate light absorbers as photoanode or photcathode materials with regard to the nanoparticle scattering properties and the size and shape dispersion of the particles. Another consideration would be to analyze the resulting excess minority carrier profiles - particularly in nanoscopic materials with a direct energy gap - and to assess under which conditions carrier transport can occur that extends beyond classical diffusive motion or carrier hopping. In microwire arrangements for solar fuels generators, ${ }^{35}$ the use of light scattering elements positioned between the wires at the bottom of the structure in close proximity to metallic electrocatalysts has been realized, and enhancements of the scattering range using less material can be envisaged. This would also allow better access of electrolyte to the catalysts and allow for enhanced bubble removal. In thin film solar cells with direct gap semiconductors, the losses due to superdiffusion should be considered explicitly. The spectral distribution at the front side will contain more light with band gap energy due to photon recycling. Also, the detailed balance calculations for theoretical efficiency of solar cells have to be modified if photon recycling is significant. This contribution can become substantial in materials where thermalized electrons 
and holes recombine radiatively. In systems where carrier transport poses a problem, carrier generation by superdiffusion of light could be used to generate carriers at a distance from the absorption site near collecting parts of a structure.

\subsection{Electrodes - Structural Aspects}

The concept of obtaining an optical Lévy flight by using scatterers that have self-similar fractal structures cannot be realized experimentally because of the size-dependence of the light scattering; larger particles would lead to Mie scattering, whereas small particles would almost not scatter in the Rayleigh limit. For electrodes, however, the surface structure and the total accessible surface area for catalysis are of decisive importance for catalytic activity and, besides metal electrodes, also semiconductor electrodes with fractal geometry have been realized. ${ }^{36-38} \mathrm{~A}$ large surface-to-volume ratio is thus generally desirable for electrochemical energy conversion devices such as combined photoelectrodes and electrocatalysts, batteries or fuel cells. Interestingly, a major input on this topic originates from biology, where allometry, i.e. the measurement of size or mass of body parts in relation to the whole body, has led to revealing insights ${ }^{39,40}$ regarding the allometric scaling law, which is formulated as

$$
Y=Y_{0} M^{b}
$$

Here, $Y$ is a biological variable (metabolic rate or life span, for example), $M$ the body mass, $b$ the scaling exponent and $Y_{0}$ depends on the kind of organism. In Euclidean geometric scaling, reflecting simple geometric constraints, the exponent $b$ should be a multiple of $1 / 3$. Scaling of area $\left(l^{2}\right)$ or volume $\left(l^{3}\right)$, for example, leads to a scaling exponent of $2 / 3$. It is found, however, that the exponent $b$ follows a $1 / 4$ behavior for properties as different as cellular metabolism, heartbeat, maximal population growth (all $b=-1 / 4$ ), blood circulation, life span, embryonic development $(b=1 / 4)$ and cross sectional areas of mammalian aortas, metabolic rates of entire organisms, cross-sectional areas of tree trunks $(b=3 / 4)$. This behavior has been connected with three unifying biological principles that obviously govern evolution by natural selection: (i) a space-filling branching structure which is fractal-like is needed to allow transport to the entire volume of an organism, (ii) the energy that is required to distribute resources down to the cellular level is minimized and (iii) the final branches of the fractal-like network is a size-invariant fixed unit. According to these principles, nature has developed methods to maximize metabolic rates by optimizing the surface areas where exchange of resources occur (for instance in organs such as the lung) and, also, the internal transport efficiency has been maximized by minimizing transport times via reduction of the distances within the network. Fractals are an optimal geometry for minimization of energy losses due to the transfer network while maximizing the effective surface area. Figure 16.14 illustrates a topological network representation adapted from 

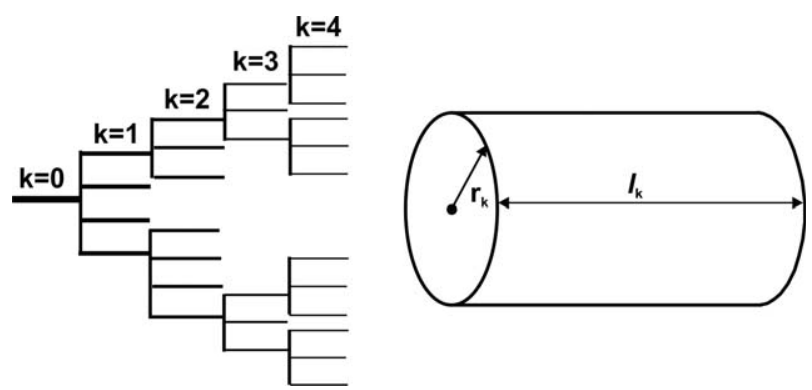

Figure 16.14 Topological representation of a biological network; $k$ : order of the respective level from large $(N=0)$ to the final fixed size (capillary, for example) at $N=k$; the right hand side shows the dimensions of a tubular element at the $k^{\text {th }}$ level (see text).

ref. 38. It has been shown that, in order to obey the principle of minimized energy dissipation in the system, the network has to be a self-similar fractal where the number of branches, $n$, increases according to $N_{\mathrm{k}}=n^{\mathrm{k}}$ when their size decreases from $N_{0}=0$ to $N$ as displayed in the figure.

Electrodes operating on the above principles would be ideal for many applications in electrochemistry and - in the context of this book - in photoelectrochemistry. This has been recognized and specifically addressed more recently. ${ }^{38}$ The case has been made that space-filling fractal structures provide the means for efficient charge transfer from an extensive surface area to a current collector. If one considers operation of electrodes, however, an additional parameter enters that has, so far, been neglected in the biological view of allometry. Besides needing to fulfill the above-mentioned three criteria for transport, the network also has to provide efficient paths for current transport and collection, which is a non-trivial problem due to the increase of specific resistivity with decreasing diameter of the current transporting element. A further challenge is the synthetic generation of such networks, which is difficult. Attempts to prepare such networks have been made using chemical vapor deposition, ${ }^{38}$ polishing, ${ }^{41}$ solid state reaction (calcination) ${ }^{42}$ or metal electrodeposition, ${ }^{43}$ to name only a few. Synthesis of fractal architectures can be achieved by "building" the structures, as in CVD, or by generating porosity (material removal) that follow a scaling law. The latter concept has been applied for light-induced fabrication of fractal structures at silicon electrodes, which show distinct azimuthal features related to the crystal orientation used. ${ }^{36,37}$ Figure 16.15 shows a typical result obtained by illumination of the silicon electrode with about $15 \%$ of 1 sun (white light, W-I lamp) for $10 \mathrm{~min}$ at anodic potentials in concentrated ammonium fluoride where oxygen evolution occurs. The structure is considerably extended over several $\mathrm{mm}$, as can be seen from the $1 \times 1 \mathrm{~mm}$ display of Figure 16.15. In principle four processes compete in the slightly alkaline $\mathrm{NH}_{4} \mathrm{~F}$ solution: ${ }^{44}$ (i) anodic light-generated hole induced oxidation, $\mathrm{O}_{2}$ evolution; (iii) oxide etching and (iv) silicon dissolution. A typical topography of such a structure (measured by a dactylograph because the depth 

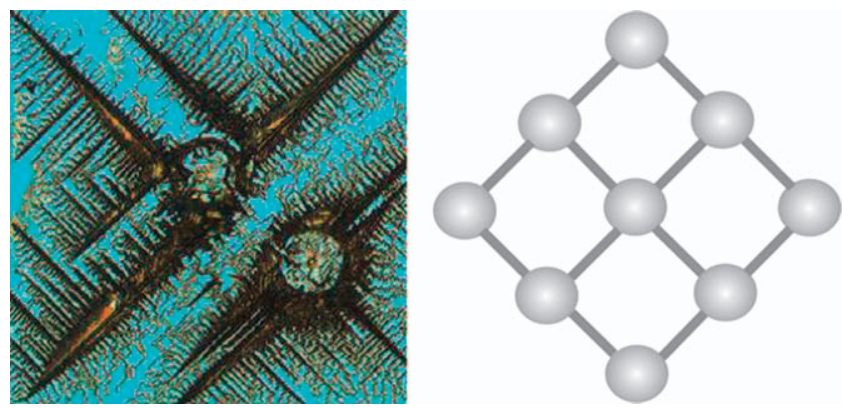

Figure 16.15 Left: light microscope image of n-Si(100) after extended photoelectrochemical etching in $40 \% \mathrm{NH}_{4} \mathrm{~F}$ at anodic potential of $\mathrm{V}=6 \mathrm{~V}(\mathrm{SCE})$. Light intensity between 4 and $8 \mathrm{mWcm}^{-2}$ (white light). Scale: $1 \times 1 \mathrm{~mm}$; right: crystal surface orientation of a (100) fcc lattice indicating the azimuthal symmetry of the fractal structures (see text).

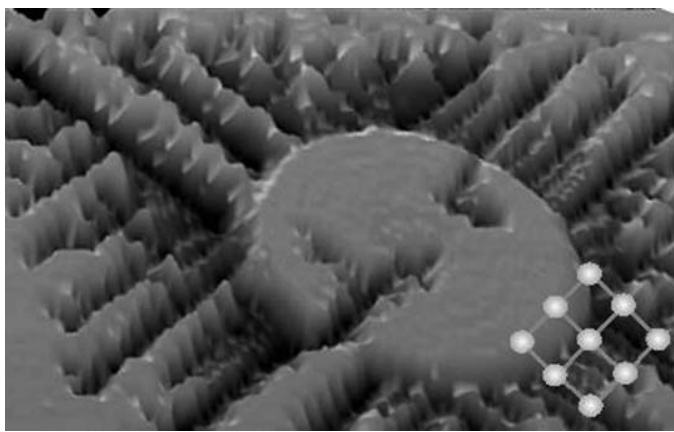

Figure 16.16 Dactylogramm of a fractal structure obtained on $\mathrm{Si}(100)$ (see crystal orientation insert) that shows depressions that are up to $6 \mu \mathrm{m}$ deep; the circular, less corroded structure in the center is about $100 \mu \mathrm{m}$ in diameter, the total picture frame is $\sim 300 \times 200 \mu \mathrm{m}$.

is too large for AFM measurements) shows depressions that are a result of crack propagation and silicon dissolution (see Figure 16.16).

The images Figures 16.15 and 16.16 show a striking resemblance to the many pictures existing of snowflakes and, particulary, of pictures of frost on a windowpane, although, instead of assembling particles from a random Brownian walk, here a dissolution pattern with rather deep trenches is observed. These features have been described using the diffusion-limited aggregation model (DLA). ${ }^{45}$ DLA is a process where the particles that are are added successively (one at a time) to a growing entity (cluster, aggregate, condensate) arrive by a random Brownian walk (classical diffusion, see also above under Lévy flights). Simulations in two dimensions show that highly ramified structures are formed that exhibit fractal geometries. For 2D and 3D clusters, the fractal exponent (or fractal dimension) is 1.7 and 2.5 , respectively. ${ }^{46}$ The similarity between crack propagation and DLA becomes more visible in the dielectric breakthrough in insulators and the corresponding observed branching. ${ }^{47}$ The basic similarity of 
crack growth to non-equilibrium processes such as $\mathrm{DLA}^{48}$ is that the probability for growth in both cases depends on the local value of a field close to the surface of the growing entity. This scalar field can be the electric potential or the concentration in the case of dielectric breakthrough or DLA, respectively. The growth probability is a function of the local value of the field or of its gradient. A complication of theoretical considerations arises from the fact that the field at each point is a function of the entire structure, which typically has a complex (fractal) geometry.

For analysis of the data on silicon electrodes, the fractal cracking approach has been followed, which reproduces the basic features by considering normal and shear stress in the surface plane assuming that Hooke's law holds. The physical origin of the stress is attributed to the well-known volume mismatch between $\mathrm{Si}$ and its oxide since it has been shown by photoelectron emission microscopy (PEEM) that the structures formed are partially oxidized (see Figure 16.17. ${ }^{49}$ The figure demonstrates that the tip and the surrounding areas exhibit different surface conditions; whereas the tip shows a signal from silicon (right hand side of Figure 16.17), the surrounding areas consist largely of oxidized surfaces. For applications in photoelectrochemical water splitting, this results in a spatially inhomogeneous distribution of electrocatalysts, where the deposition occurs on the tips but not in the surrounding areas.

In the model, the oxide-induced interfacial stress and strain results in crack propagation and development of fractal structures. It has been assumed that near edges or at tips of the structures the influence of the stress results in larger strain as atoms at tips, kink sites and edges are less coordinated to the Si lattice. Strain at a surface-near region is known to facilitate crack propagation. ${ }^{36,37}$ In the photoelectrochemical experiment for fractal structure formation, the cracking is accompanied by solvolytic attack of backbonds by water and, in the presence of holes, oxide and hydroxide formation $(\mathrm{pH} \sim 8)$ with resulting dissolution and three-dimensional etching at the cracks. The trench formation is a result of the deflection of holes to the sites where the electric field is largest and

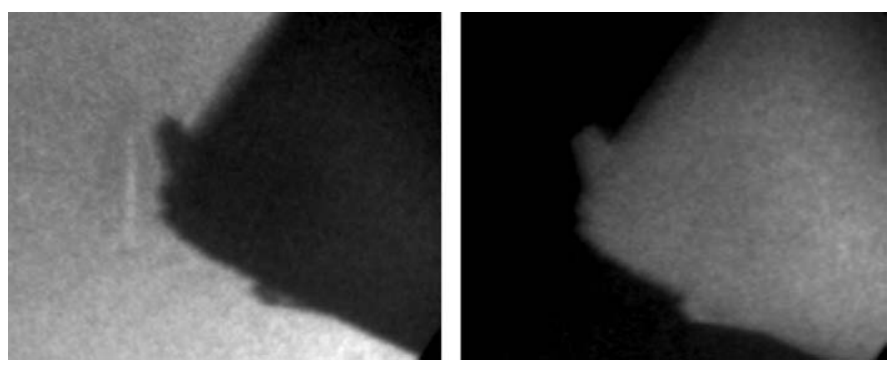

Figure 16.17 PEEM data from the inner part (a tip) of a fractal structure as shown in Figures.16.14 and 16.15. The contrast is defined as follows: left, photoelectron yield from silicon oxide, measured at a XPS bindingenergy of $104 \mathrm{eV}$; right, photoelectron yield from silicon measured for a binding energy of $99 \mathrm{eV}$. The displayed tip length is about $15 \mu \mathrm{m}$ (see text). 
where holes are consumed predominantly. Nevertheless, considering the lateral and perpendicular extension of the structures, $L_{\mathrm{p}}, L_{\mathrm{s}}$, respectivly the ratio $L_{\mathrm{p}} /$ $L_{\mathrm{s}}$ is $\sim 10^{-3}$ indicating that a $2 \mathrm{D}$ description is a good approximation.

One notes in Figures 16.15 and 16.16 that a large central area exists and that the structure development appears to initiate predominantly at the boundaries of the structure. Since substantial $\mathrm{O}_{2}$ evolution takes place in the photoelectrochemical experiment, it has been assumed that particularly pronounced oxide formation occurs at the three-phase boundary at the circumference of a not yet detached oxygen bubbles where $\mathrm{O}_{2}$ (inside the bubble), electrolyte and silicon coexist. It is known the $\mathrm{Si}$ oxidizes particularly efficiently in the presence of both oxygen and water, generating silicon oxide with a high rate and maximum thickness. The oxide formed is thought to be the seed for the spread of the fractal structure that can be seen in the figures. Figure 16.18 shows a schematic of the present model of the origin of the structure development. The arrows indicate the compressive stress due to the volume expansion of the oxide $\left(V_{\mathrm{SiO} 2} \sim 2.2 V_{\mathrm{Si}}\right)$.

For applications in water splitting structures, it would be necessary to extend the fractal geometry further into the $z$-direction (perpendicular to the surface) in order to generate well-defined fractal geometries with extremely large internal surface areas. The preparation process is scalable and occurs at room temperature; however, a method to deepen the structures has not yet been developed. Based on the experience with thin film silicon, it suffices if one creates trenches that are in the range of those observed in Figures 16.15 and 16.16. ${ }^{50}$ Actually, since some thin film Si systems operate with thicknesses down to $1.5 \mu \mathrm{m}$, one has to be consider leaving an intact Si backbone for electrode support.

Deposition of electrocatalysts can be achieved more uniformly if the material is etched to remove surface oxide. In the view of recent attempts to fabricate photovoltaic "cores" of solar fuel generators using Si-based tandem structures, the method shown here also holds promise for subsequent deposition of interlayers and a surface transition metal oxide as photoanode for realization of large-area photoelectrodes with defined geometry. A geometry that exhibits a fractal-type structure has been introduced by the Grätzel group (see also

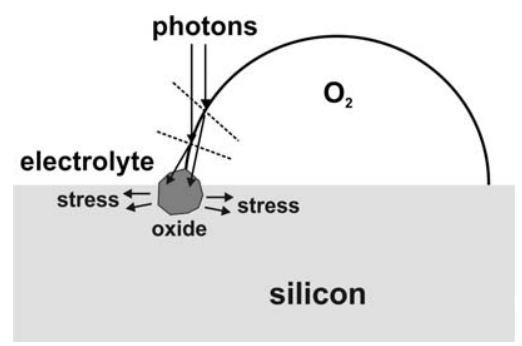

Figure 16.18 Schematic of the formation of an oxide seed for crack propagation at the circumference of an oxygen bubble; note that the bubble is an optically less dense medium compared to the electrolyte (see text). 
chapter 4$)$, where hematite $\left(\mathrm{Fe}_{2} \mathrm{O}_{3}\right)$ has been prepared with a "broccoli-type" structure $^{51}$ because the carrier diffusion lengths are so low (in the low nm range) that excess minority carrier transport to the surface is restricted to the nearsurface region (some reasoning for this electronic behavior will be given below in the next sub-section on transition metal oxides). In a ramified structure, carriers have a larger probability of reaching the surface for charge transfer than in a planar one. Since, the performance of $\mathrm{BiVO}_{4}$ is also believed to be transport limited, ${ }^{52}$ this points to (i) the need to control mesoscopic transport properties across nanoparticular boundaries and (ii) possible complexities of the energy band structure of transition metal oxides. The latter topic is addressed in the following section.

\subsection{A Note on Electronic Properties of Transition Metal Oxides}

Transition metal oxides (TMOs) are a materials class that is currently being investigated intensively for application as photoanodes in solar fuel generation systems (see chapters 4 and 5). Recent experiments on TMOs show some peculiarities of their band structure that are outlined here since they influence transport, Fermi level position, contact behavior, carrier lifetimes and optical properties, including the excess minority carrier profiles after photoexcitation. Much work has been focused on the origin of the mobility gap, particularly in the context of understanding perovskite high temperature superconductors. ${ }^{53,54}$ The origin of the energy gap can be envisaged in a simplicistic schematic as shown in Figure 16.19 for the case of a ground state configuration of the five $d$ orbitals in a crystal field of cubic symmetry. The splitting of the levels is such that their center of gravity with respect to energy is not affected by the perturbation; hence $3 E_{\mathrm{t} 2 \mathrm{~g}}+2 E_{\mathrm{eg}}=0$. Historically, the energy of the free ion ground state was set to zero, and the energetic difference between the three $t_{2 \mathrm{~g}}$ and the two $e_{\mathrm{g}}$ levels was defined as $10 \mathrm{Dq} .{ }^{55}$ The splitting of the levels is then, as shown in Figure 16.19:

$$
E_{t 2 g}=-4 D q \quad E_{t 2 g}=-4 D q \quad E_{e g}=+6 D q
$$

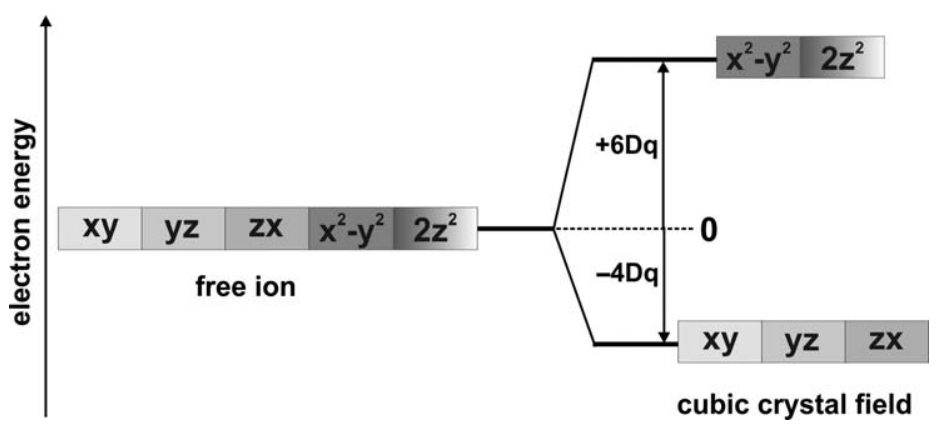

Figure 16.19 Schematic of d-band splitting in a solid with cubic crystal field (see text). 


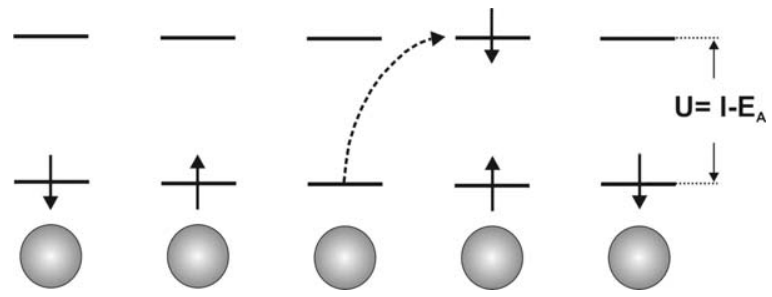

Figure 16.20 Schematic on Coulomb repulsion resulting in Mott-Hubbard gap, where the Hubbard interaction is expressed by the difference between ionization energy $I$ and electron affinity $E_{\mathrm{A}}$ (see text).

Rather recently, it has been recognized that the crystal field splitting (which is larger for tetragonal symmetries) and the influence of electron repulsion, which leads to localization (see Figure 16.20), can play a role in the determination of the energy band structure of TMOs. ${ }^{56}$ Here, we restrict ourselves to the Coulomb repulsion and related effects for the analysis of band structure properties. As this subsection is written as a note, it emphasizes specific findings without claiming generality, and the focus here is on the localization and delocalization of electrons in strongly correlated systems. Consider the simple case of a one dimensional arrangement of d-atoms, originally introduced by Hubbard, Kanamori and Gutzwiller, ${ }^{57-59}$ with Coulomb electron repulsion for placing two electrons at an atomic site within a solid (Figure 16.20).

The energy balance is written

$$
U=E\left(d^{n+1}\right)+E\left(d^{n-1}\right)-2 E\left(d^{n}\right)
$$

where, $E\left(d^{n}\right)$ is the total energy of the system of $n$ electrons in a given atom and in a given $d$-shell. The above considerations are valid for materials where the energy gap arises between the d-states of the transition metal having an intrametallic $d-d$ band gap. This is the case only for the so-called early TMOs. In that case, the occupied oxygen related $p$-bands are energetically well below the Hubbard-type d-bands. For late TMOs in the periodic table, the situation changes: the d-states are located closer to the $\mathrm{O} p$ bands such that $E(\mathrm{~d})-$ $E(\mathrm{p})<U$. As result, the lower lying Hubbard band is located energetically below the top of the $\mathrm{O} p$ band as shown for both situations in Figure 16.21.

In the latter case, the lowest excitation energy corresponds to a transition from occupied oxygen orbitals to empty $d$ states (the unoccupied upper Hubbard band) which, in the notation of Zaanen, Sawatzky and Allen ${ }^{60}$ is called a charge transfer insulator (as indicated in the figure) because the lowest excitation corresponds to an electron transfer from an $\mathrm{O}$ to a metal atom within the compound. For a single band approximation, the interaction is typically described by the Hamilton operator in the framework of second quantization $^{61,62}$

$$
H=-t \cdot \sum_{\langle i, j\rangle, \sigma} c_{i \sigma}^{+} c_{j \sigma}+U \cdot \sum_{i} n_{i \uparrow} \cdot n_{i \downarrow}
$$



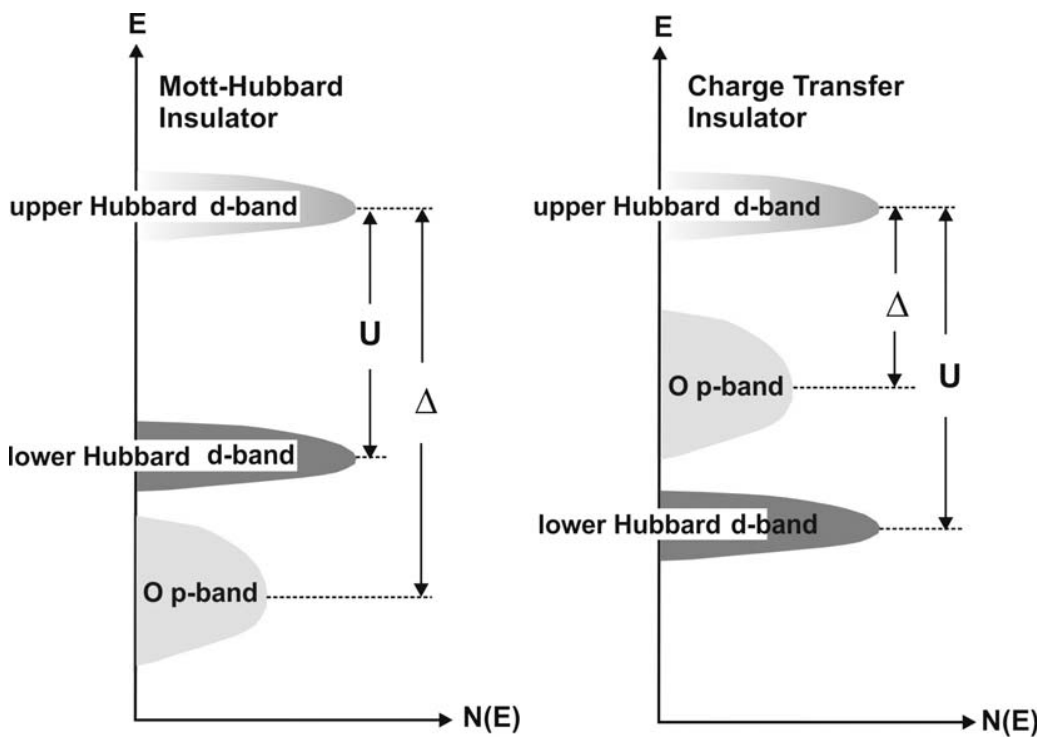

Figure 16.21 Schematic of the energy relations of d- and p- bands in Hubbard (left) and charge transfer insulators(right); $U$ : Hubbard parameter; $\Delta$ : charge transfer parameter (see text).

Here, $t$ denotes the so-called transfer integral in the framework of tight binding theory for conduction ${ }^{63}$ and $U$ is the Hubbard parameter; both are distance dependent. The symbol $c$ stands for the creation $\left(c^{+}\right)$and annihilation operator (c) of an electron at position $i$ or $j$, respectively, with spin $\sigma$. The bracket with $i, j$ denotes the nearest neighbor interaction in the lattice. The number operators $n_{i \uparrow}, n_{i \downarrow}$ describe the doubly occupied sites and the second term in equation (16.16) represents the energy for the doubly occupied single sites of the system. The charge transfer energy (first term) and the Coulomb interaction (including exchange, second term) describe the basic properties of the system. Figure 16.22 shows a highly simplistic visualization of the situation for a $1 \mathrm{D}$ chain of atoms in the tight binding approximation.

The schematic above refers to transport in a system where the low energy localized states are formed by a charge transfer band. Accordingly, the extended states (see upper level) form Bloch-type bands with delocalized electrons. The inner shells are described by localized states where intersite tunneling can occur which delocalizes these electrons to some extent. In the lowest part of Figure 16.22, the inner structure of the atom and the orbitals is neglected and electrons are considered that hop from site to site. Hopping between more distant sites can be neglected in this approximation, where the intersite tunneling depends on the distance and the localized shell's radial wave function decays exponentially with distance. The corresponding Hamiltonian can be described by (see also equation (16.16))

$$
H=-\sum_{\langle i, j\rangle, \sigma} t_{i j} c_{i \sigma}^{+} c_{j \sigma}
$$




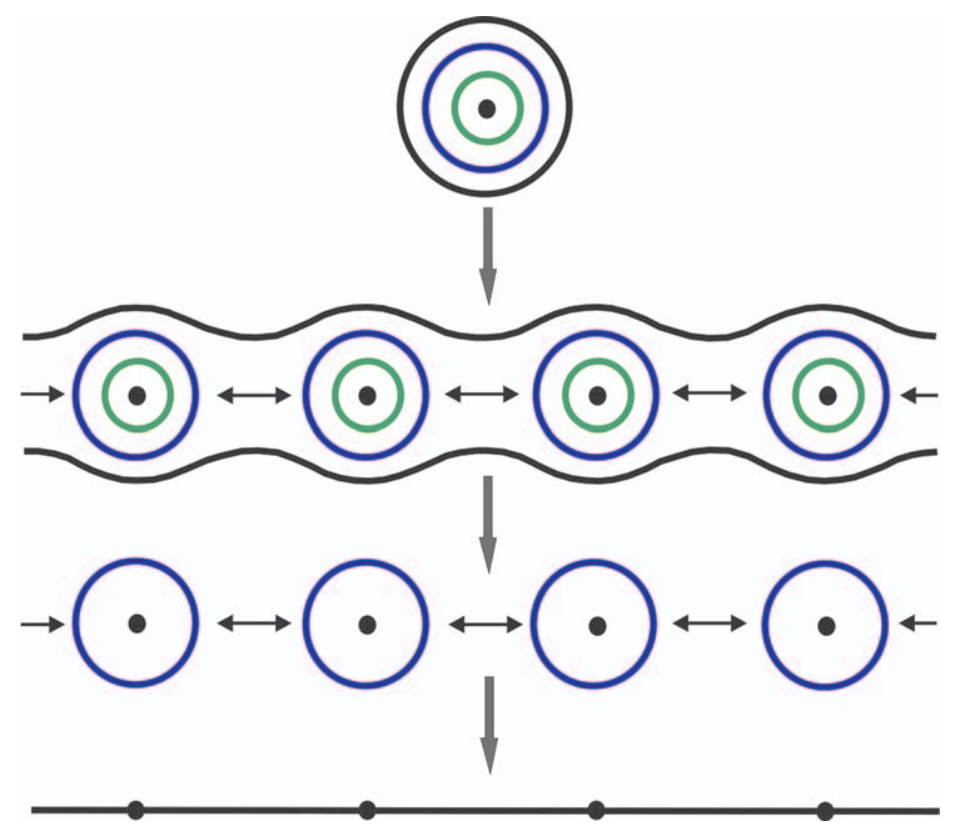

Figure 16.22 Simplified representation of tight binding descriptions of bonding. Upper level: single atom with core (black) and electrons in different (black and color) orbitals. Below: upon decreased distance in a solid, outer electrons (black circles) become itinerant, those in inner shells (dark blue, green) remain localized at the site of the atom, but, as indicated by the arrows, can tunnel with a certain probability to another adjacent colored orbital; third level. One considers only the behavior of the electrons in the more localized shells only and their role on the low energy electronics of the system. Lowest level: the electron behavior is given by localized electrons (at the position of the lattice sites) that move by hopping from on site to another (see text).

where $t_{\mathrm{ij}}=t_{\mathrm{ji}}$, and $t_{\mathrm{ii}}=0$. The matrix element for tunneling, $t_{\mathrm{ij}}$, describes the wave function overlap and the tunneling probability of an electron from site $i$ to site $j$ :

$$
t_{i j}=\int \varphi^{*}\left(r-R_{i}\right) \cdot \varphi\left(r-R_{j}\right) d^{3} r
$$

The hopping Hamilton operator in equation (16.17) sums over all hopping processes: the operators $c_{\mathrm{i}}^{+}$and $c_{\mathrm{j}}$ destroy an electron at site $j$ and recreate the electron at the adjacent site $i$ (see also above text).

The energy band structure of the TMOs can be assessed by resonant photoemission, ${ }^{64}$ inverse photoemission ${ }^{65}$ and x-ray absorption spectroscopy. ${ }^{66}$ For the hematite $\left(\mathrm{Fe}_{2} \mathrm{O}_{3}\right)$ and titania $\left(\mathrm{TiO}_{2}\right)$, which are investigated intensively for solar fuel and solar photovoltaic applications, the energy band structure resulting from recent measurements ${ }^{67}$ is presented schematically in Figures 16.23 and 16.24. 


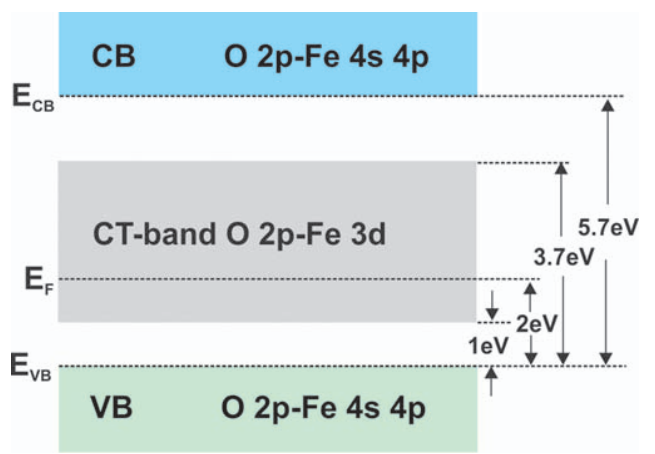

Figure 16.23 Energy band structure of hematite $\left(\alpha-\mathrm{Fe}_{2} \mathrm{O}_{3}\right)$ as determined from resonant photoemission. ${ }^{64} \mathrm{CT}$-band: charge transfer band; VB, CB: conduction- and valence band; $E_{\mathrm{VB}}, E_{\mathrm{CB}}$ : valence band maximum and conduction band minimum, respectively; $E_{\mathrm{F}}$, Fermi level; the optical gap of $2 \mathrm{eV}$ is located between Bloch-like valence band and charge transfer states (see text).

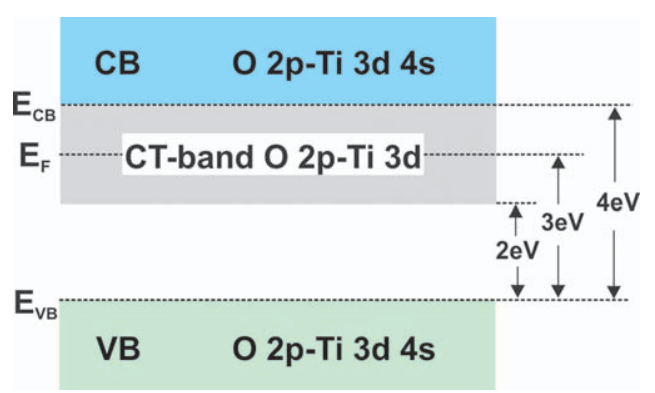

Figure 16.24 Energy band structure for $\mathrm{TiO}_{2}$; symbols as in Figure 16.22. Note that in both cases shown in the above figures, the Fermi level is positioned within the charge transfer band (see text).

For $\mathrm{TiO}_{2}$, the following transitions have been identified by optical methods. Photoluminescence shows transition energies of $2.0 \mathrm{eV}$ and $2.2-2.4 \mathrm{eV}$. Ellipsometry shows transitions at 3.2 and $4.2 \mathrm{eV}$; this is further supporting evidence of the energy scheme shown above. For hematite, the $2 \mathrm{eV}$ transition is mostly known and considered as the energy gap. ${ }^{68}$ The presence of Hubbard- or charge transfer bands within a Bloch-state energy gap explains the problems in electron transport encountered in these materials. It has to be shown in future work how one can control the position of the Fermi level position, the formation and extension of space charge layers and how to improve transport properties. Since the development of stable and efficient photoanodes is a crucial component of light-induced water splitting, such research will be particularly beneficial for this goal. 


\subsection{Looking Back}

It is always difficult to assemble a book like the present one in such a way that the coverage is comprehensive and strongly interlinked. Therefore, in the concluding paragraphs of this final chapter, we try to pull some of the ideas together by looking at trends that can be discerned from the preceding chapters as well as from the current literature. The first feeling that the editors gained from colleagues working on this book is a palpable sense of excitement about the science involved, a feeling that this time we are really getting closer to the final goal. At the same time, enthusiasm for the basic science has to be tempered by an increasing awareness that as research in the laboratory is translated into working devices, considerations of cost and competitiveness need to guide the selection of technologies for scale up and testing. In Chapter 1, Parkinson and Turner take a hard look at the potential contribution of photoelectrochemical energy conversion and storage to the global energy future. They argue convincingly that technoeconomic analysis indicates that the rather comfortable assumption that $10 \%$ solar to hydrogen efficiency is adequate for a viable technology is not justified. Instead a much more demanding efficiency of $15 \%$ or more, combined with a lifetime in excess of 20 years, will be required. These are challenging targets that deserve more widespread discussion. Parkinson and Turner also take the - possibly controversial - view that the photoelectrochemical reduction of $\mathrm{CO}_{2}$ is really an approach of last resort; one that only makes sense when virtually all carbon-based power generation has been replaced by renewable alternatives. Of course, this conclusion should not deter us from continuing to search for ways of mimicking photosynthesis, and in chapter 11, Rajeshwar and his colleagues give an excellent overview of some of the exciting science involved in trying to reduce $\mathrm{CO}_{2}$, while Schouten and Koper give a very comprehensive account of theoretical aspects of $\mathrm{CO}_{2}$ reduction in chapter 12. The point about Parkinson and Turner's discussion is that is draws attention to the importance of realistic assessments of the potential of different technologies. All too often, in the introduction to scientific papers one reads bland statements about a supposedly "low cost technology" that are not backed up by any citations of a corresponding technoeconomic analysis.

Although the majority of the book deals with basic science, the importance of engineering in the design of practical water splitting systems is highlighted by McKone and Lewis in chapter 3. Many of the electrochemical engineering issues discussed in this chapter are similar to those involved in the design of commercial electrolyzers for hydrogen or chlorine generation: mass transport, bubble formation and safe separation of the products. It certainly makes sense to involve electrochemical engineers at an early stage of device development in order to be able to define a complete system that can be subjected to technoeconomic analysis. The overall design becomes more complex when a photoelectrochemical cell is combined with an integrated solar cell in the kind of tandem system advocated by Sivula and Grätzel in chapter 4. The matching of 
the two active light absorbing components will clearly be critical, and of course all of the preceding engineering considerations apply as well. This leads us to consider the trade-off between complexity and performance. If Parkinson and Turner are right, many of the photoelectrochemical systems currently being studied may not make it past a critical cost analysis. Only ultra-low cost systems can afford lower efficiencies, and it is an open question whether the kind of particulate systems described by Maeda and Domen in chapter 5 may ultimately fall into this category. If, on the other hand, very high efficiency systems really are the only option, then perhaps the group II-nitride systems discussed by Collazo and Dietz in chapter 8 or the III-V thin film structures reviewed in chapter 9 by Hannappel et al. may prove to be the most viable way forward. Nevertheless, materials science aspects of water splitting are likely to remain important. Some idea of the richness of the existing materials landscape is given by Fiechter in chapter 7, and the potential of rapid screening methods to identify promising new materials is clear from the survey given in chapter 6 by Bard and co-workers. At the same time, it is often helpful to return to Nature for new ideas and inspiration, as is clear from chapter 14, where Ostroumov et al. examine different light harvesting strategies.

The role of computational methods in the development of water splitting systems has already been mentioned. The general relevance of computational studies in energy materials is nicely illustrated by a recent overview by Catlow et $a .^{68}$, and more specific examples are given in the present book by Hellman in chapter 10. Sometimes chemical instinct is not enough to ensure progress in such a complex field as light-driven water splitting, and it seems likely that computational studies will make and increasingly important contribution to a range of relevant topics, including new materials, catalyst as well as aspects of kinetics and mechanisms. Here, as always, the link between theory and experiment will be crucial. Many of the recent suggestions from computational work for routes to performance enhancement remain to be tested in practice, and in the future will be essential to establish much closer cooperation between theoretical and experimental groups in order to pinpoint key issues and to guide research directions.

\subsection{Progress Towards Light Driven Water Splitting}

In these final sections, we want to take stock of the current situation and review progress. Forty years have passed since the publication of Fujishima and Honda's Nature much-cited paper, ${ }^{69}$ but in spite of a resurgence of interest, we are still some way from realizing the goal of a stable low cost system for splitting water efficiently using sunlight. One has to ask why progress has been so slow. The answer is largely connected with the complexity of the water splitting reaction, particularly the half reaction leading to molecular oxygen. The four electron/4 proton oxidation of two molecules of water to form $\mathrm{O}_{2}$ is achieved in higher plants by Photosystem II, in which the four electrons are transferred to two plastoquinone molecules via the manganese centre, which contains 4 manganese atoms, a bound calcium ion and a tyrosine radical. 
The $\mathrm{P} 680^{+}$formed by the first electron transfer quenching of the excited $\mathrm{P} 680^{*}$ state is a strong oxidant, and plants repair of the Photosystem II centre several times a day. This places into context the extraordinarily ambitious goal of designing a water splitting system that will be stable for decades. Even oxides like $\mathrm{TiO}_{2}$ undergo slow photocorrosion, particularly in acidic solutions, and generally other compound semiconductors are much less stable than transition metal oxides. The difficulty of preventing normal corrosion of a twenty year time scale is a fact of everyday life, and water splitting systems are no exception. The concept of self-repair is a feature of the cobalt-based oxygen evolving catalyst discovered by Nocera et al. ${ }^{70}$ and it may be possible to develop selfrepairing components for water splitting systems as a way of overcoming basic issues of thermodynamic instability.

The complexity of the light-driven oxygen evolution reaction presents an enormous challenge to theory and practice. It is clear that the 4-hole reaction at n-type photoanodes must proceed via a series of surface bound intermediates, but even in the case of model photoanode systems such as rutile, details of the oxygen evolution mechanism remain controversial ${ }^{71-73}$ in spite of spectroscopic evidence (FTIR) for the formation of peroxo or superoxo species. In the case of hematite photoanodes, UV-visible spectroscopic evidence also suggests formation of a surface peroxo or superoxo intermediate, but the exact identity of the species is unclear. ${ }^{74}$ In both cases, the first step is generally thought to involve the trapping of a hole at surface site. The progressive refinement of computational approaches ${ }^{75-78}$ is providing useful guidance regarding possible pathways for the oxygen evolution reaction, but much remains to be done to obtain reliable quantitative results that can be compared with experiment. Generally, computational approaches deal with well-defined surfaces corresponding to particular crystal orientations, but as far as experimental studies are concerned, little is known about the influence of surface structure and orientation on the mechanism and kinetics of light-driven water splitting. One of the problems in this respect is that some of the best photoanode materials such as hematite have been nanostructured to enhance performance. The increase in complexity makes it difficult to apply conventional models for the semiconductor/electrolyte interface, and as a consequence extraction of kinetic information from photoelectrochemical experiments can be difficult. Probably the best way forward for the quantitative study of such systems is to use timeresolved in situ spectroscopic techniques. ${ }^{79}$

The search for new materials for solar water splitting is being pursued using combinatorial $^{80-84}$ and also computational ${ }^{84-87}$ approaches. Here one of the main problems is that optimization of a large range of solid-state, optical and chemical properties is generally necessary in order to obtain an efficient and stable $^{88}$ photoanode or photocathode material. The pathway from identifying promising candidates to development of a viable stable water splitting electrode is generally time-consuming, but the necessary research input is now becoming more readily available as water splitting moves towards centre stage, partially displacing other heavily researched areas such as dye-sensitized solar cells. A measure of the enormous increase in research effort is provided by figures for 
the Joint Center for Artificial Photosynthesis, the world's largest research programme dedicated to the development of an artificial solar fuel generation technology: a budget of $\$ 122 \mathrm{M}$ over 5 years, a workforce of over 130 , 8 research divisions and 5 scientific user facilities. In his State of the Union Address in 2011, Barack Obama put it in these terms: "We're issuing a challenge. We're telling America's scientists and engineers that if they assemble teams of the best minds in their fields and focus on the hardest problems in clean energy, we'll fund the Apollo projects of our time ... At the California Institute of Technology, they're developing a way to turn sunlight and water into fuel for our cars ... We need to get behind this innovation." The signs are that the next decade will take us much closer towards the ultimate goal of splitting water to produce solar fuels, and we hope that this book will be a source to inspire new ideas about how to achieve this.

\section{Acknowledgements}

This material is based upon work performed by the Joint Center for Artificial Photosynthesis, a DOE Energy Innovation Hub, supported though the Office of Science of the US Department of Energy under Award No. DE-SC0004993. HJL acknowledges additional financial support from DFG, grant No. Le 11924. The authors are grateful to C. Pettenkofer and M. Lublow for experiments regarding PEEM and fractal Si formation.

\section{References}

1. H. Raether, Surface Plasmons on Smooth and Rough Surfaces and on Gratings, Springer Tracts in Modern Physics, Springer, Berlin, New York, 1988.

2. N. J. M. Horing, in Introduction to Complex Plasmas, Springer Ser. Atomical, Optical and Plasma Phys., Vol. 59, ed. M. Bonitz, N. Horing, P. Ludwig, Springer Heidelberg, 2010, pp. 109-134.

3. S. M. Sze, Semiconductor Devices, Wiley and Sons, 1980.

4. R. H. Ritchie, Surf. Sci., 1973, 34, 1-19.

5. R. Kötz, H. J. Lewerenz and E. Kretschmann, Phys. Lett. A., 1979, 70, 452-454.

6. J. K. Sass and H. J. Lewerenz, J. de Physique, 1977, 38, 277-284.

7. H. Neff, J. K. Sass, H. J. Lewerenz and H. Ibach, J. Phys. Chem., 1980, 84, 1135-1139.

8. Y. Hori, H. Wakebe, T. Tsukamoto and O. Koga, Electrochim. Acta, 1994, 39, 1833-1839.

9. K. A. Willets and R. P. Van Duyne, Annu. Rev. Phys. Chem., 2007, 58, 267-297.

10. T. Vo-Dinh, Trends in Analytical Chem., 1998, 17, 557-582.

11. D. K. Roper, W. Ahn and M. Hoepfner, J. Phys. Chem. C, 2007, 111, 3636-3641. 
12. M. Lublow, K. Skorupska, S. Zoladek, P. J. Kulesza, T. Vo-Dinh and H. J. Lewerenz, Electrochem. Comm., 2010, 12, 1298-1301.

13. R. Solarska, A. Krolikowska and J. Augustynski, Angew. Chem. Internat. Ed., 2010, 49, 780-783.

14. X. Zhang, Y. L. Chen, R.-S. Liu, D. P. Tsai and Rep. Prog, Phys., 2013, 76, 046401 .

15. D. B. Ingram and S. Linic, J. Am. Chem. Soc., 2011, 133, 5202-5205.

16. K. Awazu, M. Fujimaki, C. Rockstuhl, J. Tominaga, H. Murakami, Y. Ohki, N. Yoshida and T. Watanabe, J. Am. Chem. Soc., 2008, 130, 1676-1680.

17. H. J. Lewerenz: Photons in Natural and Life Sciences - an Interdisciplinary Approach, Springer Heidelberg, New York, 2012.

18. S. Jang S, M. D. Newton and R. J. Silbey, Phys. Rev. Lett., 2004, 92, 218301.

19. G. D. Scholes and G. R. Fleming, J. Phys. Chem. B, 2000, 104, 1854.

20. D. L. Dexter, J. Chem. Phys., 1953, 21, 836.

21. B. O'Regan and M. Grätzel, Nature, 1991, 353, 737-740.

22. M. Graetzel and J. Photochem., Photobio. C: Photochem. Rev., 2003, 4, 145-153.

23. P. Andrew and W. L. Barnes, Science, 2004, 306, 1002-1005.

24. P. Barthelemy, J. Bertolotti and D. S. Wiersma, Nature, 2008, 453, 495498.

25. M. Burresi, V. Radhalakshmi, R. Savo, J. Bertolotti, K. Vynck and D. S. Wiersma, Phys. Rev. Lett., 2012, 108, 110604.

26. R. Brown, Phil. Mag., 1828, 4, 161-173.

27. A. Einstein, Ann. d. Phys., 1905, 17, 549.

28. A. Einstein: Investigations of the Theory of Brownian Movement, Dover, 1956.

29. J. Bertolotti, Ph D thesis, University of Florence, 2007.

30. G. Polya, Math. Zeitschr., 1920, 8, 171-181.

31. Z. Cheng and R. Savit, J. Math. Phys., 1987, 28, 592.

32. R. Patel and R. Mehta, J. Nanophoton., 2012, 6, 069503.

33. S. Luryi and A. Subashiev, Internat. J. High Speed Electr. Syst., 2012, 21, 1250001.

34. S. Luryi, O. Semyonov, A. Subashiev and Z. Chen, Phys. Rev. B, 2012, 86, 201201.

35. E. L. Warren, J. R. McKone, H. A. Atwater, H. B. Gray and N. S. Lewis, Energy \& Environm. Sci., 2012, 5, 9653.

36. M. Lublow and H. J. Lewerenz, Electrochem. Solid-State Letters, 2007, 10, C51-C55.

37. M. Lublow and H. J. Lewerenz, Electrochim. Acta, 2009, 55, 340-349.

38. B. Y. Park, R. Zaouk, C. Wang and M. J. Madou, J. Electrochem. Soc., 2007, 154, P1-P5.

39. G. B. West, J. H. Brown and B. J. Enquist, Science, 1997, 276, 122-124.

40. G. B. West, J. H. Brown and B. J. Enquist, Science, 1999, 284, 1677-1679. 
41. V. Lakshminarayanan, R. Srinivasan, D. Chu and S. Gilman, Surf. Sci., 1977, 329, 44-51.

42. A. Eftekhari, Electrochim. Acta, 2002, 47, 4347-4350.

43. C.-P. Chen and J. Jorné, J. Electrochem. Soc., 1990, 137, 2047.

44. M. Letilly, K. Skorupska, H. J. Lewerenz, J. Phys. Chem. C, in print.

45. T. A. Witten and L. M. Sander, Phys. Rev. B, 1983, 27, 5686-5697.

46. O. Malcai, D. A. Lidar, O. Biham and D. Avnir, Phys. Rev. E, 1997, 56, 2817-2828.

47. L. Niemeyer, L. Pietronero and H. Wiesmann, Phys. Rev. Lett., 1984, 52, 1033-1036.

48. Y. Termonia and P. Meakin, Nature, 1986, 320, 429.

49. M. Lublow, W. Bremsteller and C. Pettenkofer, J. Electrochem. Soc., 2012, 159, D333-D339.

50. K. Yamamoto, A. Nakajima, M. Yoshimi, T. Sawada, S. Fukuda, T. Suezaki, M. Ichikawa, Y. Koi, M. Goto, T. Meguro, T. Matsuda, M. Kondo, T. Sasaki and Y. Tawada, Solar Energy, 2004, 77(2004), 939-949.

51. A. Kay, I. Cesar and M. Graetzel, J. Am. Chem. Soc., 2006, 128, 15714 15721.

52. F. F. Abdi, N. Firet and R. v.d. Krol, ChemCatChem, 2013, 5, 490-496.

53. A. P. Goncalves, I. C. Santos, E. B. Lopes, R. T. Henriques, M. Almeida and M. O. Figueiredo, Phys. Rev. B, 1988, 37, 7476-7481.

54. E. Dagotto, Rev. Mod. Phys., 1994, 66, 763-84.

55. F. J. Morin, Bell Syst. Tech. Journ., 1958, 07, 1047-1084.

56. A. I. Poteryaev, M. Ferrero, A. Georges and O. Parcollet, Phys. Rev. B, 2008, 78, 045115.

57. J. Hubbard, Proc. R. Soc. London A, 1963, 276, 238.

58. J. Kanamori, Prog. Theor. Phys., 1963, 30, 275.

59. M. C. Gutzwiller, Phys. Rev. Lett., 1963, 10, 159.

60. J. Zaanen, G. A. Sawatzky and J. W. Allen, Phys. Rev. Lett., 1985, 55, 418.

61. H. P. Paar: An Introduction to Advanced Quantum Physics, Wiley \& Sons, 2010.

62. F. H. L. Essler, H. Frahm, F. Goehmann, A. Kluemper, V. E. Korepin: The One Dimensional Hubbard Model, Cambridge Univ. Press, Cambridge, New York, 2005.

63. P. A. Cox: Transition Metal Oxides: An Introduction to Their Electronic Structure and Properties, Clarendon Press, Oxford, 1992.

64. A. E. Bocquet, T. Mizokawa, K. Morikawa, A. Fujimori, S. R. Barman, K. Maiti, D. D. Sarma, Y. Tokura and M. Onoda, Phys. Rev. B, 1996, 53, 1161-1170.

65. R. Zimmermann, P. Steiner, R. Claessen, F. Reinert, S. Hüfner, P. Blaha and P. Dufek, J. Phys, Condens. Matter, 1999, 11, 1657.

66. T. Saitoh, A. E. Bocquet, T. Mizokawa, H. Namatame, A. Fujimori, M. Abbate, Y. Takeda and M. Takano, Phys. Rev. B, 1995, 51, 1394213951.

67. D. Schmeisser, to be published. 
68. C. R. A. Catlow, Z. X. Guo, M. Miskufova, S. A. Shevlin, A. G. H. Smith, A. A. Sokol, A. Walsh, D. J. Wilson and S. M. Woodley, Philosophical Transactions of the Royal Society a-Mathematical Physical and Engineering Sciences, 2010, 368, 3379-3456.

69. A. Fujishima and K. Honda, Nature, 1972, 238, 37-38.

70. M. W. Kanan and D. G. Nocera, Science, 2008, 321, 1072-1075.

71. R. Nakamura and Y. Nakato, J. Am. Chem. Soc., 2004, 126, 1290-1298.

72. P. Salvador, Prog. Surf. Sci., 2011, 86, 41-58.

73. R. Nakamura, T. Okamura, N. Ohashi, A. Imanishi and Y. Nakato, J. Am. Chem. Soc., 2005, 127, 12975-12983.

74. C. Y. Cummings, F. Marken, L. M. Peter, K. G. U. Wijayantha and A. A. Tahir, J. Am. Chem. Soc., 2012, 134, 1228-1234.

75. J. Rossmeisl, Z. W. Qu, H. Zhu, G. J. Kroes and J. K. Norskov, J. Electroanal. Chem., 2007, 607, 83-89.

76. H. Dau, C. Limberg, T. Reier, M. Risch, S. Roggan and P. Strasser, Chemcatchem, 2010, 2, 724-761.

77. A. Valdes, Z. W. Qu, G. J. Kroes, J. Rossmeisl and J. K. Norskov, Journal of Physical Chemistry C, 2008, 112, 9872-9879.

78. P. Liao and E. A. Carter, Chem. Soc. Rev., 2013, 42, 2401-2422.

79. A. J. Cowan and J. R. Durrant, Chem. Soc. Rev., 2013, 42, 2281-2293.

80. M. Woodhouse and B. A. Parkinson, Chem Soc Rev, 2009, 38, 197-210.

81. W. Liu, H. Ye and A. J. Bard, Journal of Physical Chemistry C, 2010, 114, 1201-1207.

82. S. P. Berglund, H. C. Lee, P. D. Nunez, A. J. Bard and C. B. Mullins, PCCP, 2013, 15, 4554-4565.

83. J. M. Gregoire, C. Xiang, S. Mitrovic, X. Liu, M. Marcin, E. W. Cornell, J. Fan and J. Jin, J. Electrochem. Soc., 2013, 160, F337-F342.

84. K. J. Young, L. A. Martini, R. L. Milot, R. C. Snoeberger, III, V. S. Batista, C. A. Schmuttenmaer, R. H. Crabtree and G. W. Brudvig, Coord. Chem. Rev., 2012, 256, 2503-2520.

85. I. E. Castelli, D. D. Landis, K. S. Thygesen, S. Dahl, I. Chorkendorff, T. F. Jaramillo and K. W. Jacobsen, Energy \& Environmental Science, 2012, 5, 9034-9043.

86. I. E. Castelli, T. Olsen, S. Datta, D. D. Landis, S. Dahl, K. S. Thygesen and K. W. Jacobsen, Energy \& Environmental Science, 2012, 5, 5814-5819.

87. A. Valdes, J. Brillet, M. Graetzel, H. Gudmundsdottir, H. A. Hansen, H. Jonsson, P. Kluepfel, G.-J. Kroes, F. Le Formal, I. C. Man, R. S. Martins, J. K. Norskov, J. Rossmeisl, K. Sivula, A. Vojvodic and M. Zach, PCCP, 2012, 14, 49-70.

88. S. Chen and L.-W. Wang, Chem. Mater., 2012, 24, 3659-3666. 